\title{
A critical role for cellular inhibitor of protein 2 (clAP2) in colitis-associated colorectal cancer and intestinal homeostasis mediated by the inflammasome and survival pathways
}

\author{
M Dagenais ${ }^{1}$, J Dupaul-Chicoine ${ }^{1}$, C Champagne ${ }^{2}$, A Skeldon ${ }^{1}$, A Morizot ${ }^{2}$ and M Saleh ${ }^{1,2,3}$
}

Cellular inhibitors of apoptosis proteins (cIAPs) are critical arbiters of cell death and key mediators of inflammation and innate immunity. clAP2 is frequently overexpressed in colorectal cancer and in regenerating crypts of ulcerative colitis patients. However, its corresponding functions in intestinal homeostasis and underlying mechanisms in disease pathogenesis are poorly understood. We found that mice deficient in clAP2 exhibited reduced colitis-associated colorectal cancer tumor burden but, surprisingly, enhanced susceptibility to acute and chronic colitis. The exacerbated colitis phenotype of cIAP2-deficient mice was mediated by increased cell death and impaired activation of the regenerative inflammasome-interleukin-18 (IL-18) pathway required for tissue repair following injury. Accordingly, administration of recombinant IL-18 or pharmacological inhibition of caspases or the kinase RIPK1 protected cIAP2deficient mice from colitis and restored intestinal epithelial barrier architecture. Thus, CIAP2 orchestrates intestinal homeostasis by exerting a dual function in suppressing cell death and promoting intestinal epithelial cell proliferation and crypt regeneration.

\section{INTRODUCTION}

Inflammation is a protective physiological response elicited to fend off invading microorganisms, repair damaged tissues, and restore homeostasis. However, when overexuberant, overt inflammation is deleterious, leading to tissue destruction, partly through induction of cell death. Chronic inflammation is an important pathological component in a number of diseases including colorectal cancer (CAC). This is best illustrated in colitis-associated CAC developing in inflammatory bowel disease (IBD) patients. ${ }^{1}$ Although the etiology of IBD remains unknown, it is thought to arise because of loss of tolerance to the gut microbiota. ${ }^{2}$ Genome-wide association studies have implicated several pathways in IBD, including among others microbial sensing, epithelial barrier integrity, cell death, and mucosal defenses. ${ }^{3}$ Others and we have previously shown that innate immunity is critical in maintaining intestinal homeostasis by triggering tissue repair following damage. Paradoxically, it is also evident that excessive activation of the innate immune response contributes to colitis and tumorigenesis. ${ }^{4}$ Tightly linked to innate immunity is cell death, also pathogenic in IBD. Accumulating evidence implicates both apoptosis and a novel form of regulated necrosis termed necroptosis in intestinal chronic inflammation and pathology. ${ }^{5} \mathrm{~A}$ key inducer of both cell death forms is the proinflammatory cytokine tumor necrosis factor- $\alpha(\mathrm{TNF} \alpha)$ that exerts important pathological effects in IBD. Indeed, anti-TNF $\alpha$ monoclonal antibody therapy is currently the main biological treatment for IBD patients. ${ }^{6}$

TNF signaling results in two antagonistic outcomes, cell survival or cell death, and cell-fate decision is determined at the level of the cellular inhibitor of apoptosis proteins 1 and 2 (cIAP1 and cIAP2). ${ }^{7}$ Although originally posited to inhibit apoptotic caspases directly through interference with their catalysis, these E3 ubiquitin ligases act indirectly through ubiquitination of the serine/threonine kinase RIP1 (RIPK1), promoting the formation of a prosurvival signaling complex

${ }^{1}$ Department of Biochemistry, McGill University, Montréal, Québec, Canada. ²Department of Medicine, McGill University, Montréal, Québec, Canada and ${ }^{3}$ Department of Microbiology and Immunology, McGill University, Montréal, Québec, Canada. Correspondence: M Saleh (maya.saleh@mcgill.ca) 
downstream of death receptor engagement. The scaffolding function of RIP1, and not its kinase activity, is critical in preventing cell death. ${ }^{9,10}$ The subsequent recruitment of the IKK complex to RIP1 induces a classical proinflammatory nuclear factor (NF)- $\kappa B$ response, also favoring cell survival. ${ }^{7}$ RIP1 deubiquitination, by inhibition of the cIAPs or through deubiquitinase activity, causes its relocalization to a DISC (death-inducing signaling complex) where it interacts with FADD and mediates the activation of caspase- 8 . In addition to inducing apoptosis, caspase- 8 curbs the formation of a destructive and highly inflammatory RIP1/RIP3 necrosome complex. ${ }^{11}$ Ablation of FADD or caspase- 8 results in exacerbated necrosome activity that leads to embryonic lethality ${ }^{12-17}$ or tissue destruction and inflammatory pathology in adult tissues. ${ }^{18-20}$ However, although apoptosis and necroptosis are inherently antagonistic, they are not mutually exclusive and can occur simultaneously in infectious and inflammatory diseases. ${ }^{21,22}$ Whether the pathological consequences of necrosome activation are solely mediated through necrotic cell death or alternatively through hyperactivation of specific inflammatory pathways is currently debated. ${ }^{23}$ Consistent with a necroptosis-independent function of RIP1 and RIP3 in inflammation, Kang et al. ${ }^{24}$ reported that the necrosome amplified Nlrp3 inflammasome activation in caspase-8-deficient dendritic cells. Conversely, caspase-8 was reported to mediate the priming and activation of the Nlrp3 inflammasome ${ }^{25}$ highlighting the intricate interplay between apoptosis, necroptosis, and inflammasome pathways.

The cIAPs inhibit both apoptosis and necroptosis by curbing exuberant RIP kinase activity and pathological responses to death ligands including TNF $\alpha$. Whereas cIAP1 $\left(\mathrm{Birc}^{-/-}\right)$- or cIAP2 $\left(\right.$ Birc $\left.^{-/-}\right)$-deficient mice are viable, ablation of both genes results in embryonic lethality that is delayed to shortly after birth through codeletion of Tnfr1, Ripk1, or Ripk $3 .^{26}$ The individual contribution of each of the cIAPs to regulating cell death in adult tissues is beginning to emerge. We have recently reported that cIAP2, a NF- $\mathrm{BB}$-inducible member of the IAP family, is required to protect against pulmonary necrosis following influenza A virus infection. ${ }^{22}$ Notably, other IAPs, including cIAP1 and XIAP (X-linked inhibitor of apoptosis protein), do not compensate for cIAP2 loss in this context in conferring protection to the lung. ${ }^{22}$ Besides their critical role as arbiters of cell death, the cIAPs are important regulators of innate immunity. They are essential for cytokine and chemokine production following NOD1 and NOD2 stimulation, ${ }^{27}$ mediate the activation of mitogen-activated protein kinase pathways downstream of Toll-like receptor- $4,{ }^{27,28}$ and are required for efficient activation of the inflammasome. ${ }^{29}$

Guided by the structural basis of SMAC (second mitochondria-derived activator of caspases) binding to, and inhibition of the IAPs, a number of IAP antagonists (also referred to as SMAC mimetics (SMs)) were developed as potential anticancer agents. The use of these agents unraveled unexpected functions of the cIAPs in noncanonical NF- $\kappa \mathrm{B}$ activation. Although they triggered apoptosis of cancer cells in vitro through depletion of cIAP1, cIAP2, and XIAP, cell death occurred independently of caspase-3 derepression but was mediated indirectly via the induction of a TNF $\alpha$ autocrine loop. ${ }^{30-32}$ These antagonists are currently in clinical trials as anticancer agents. ${ }^{33}$ However, the exact mechanisms by which IAP depletion might curtail cancer development and the potential side effects of these compounds in various cancers have yet to be studied in vivo. cIAP2 is upregulated in regenerating colonocytes in ulcerative colitis patients. ${ }^{34}$ Furthermore, it is overexpressed in CRC and is a predictive prognostic marker of CRC recurrence after chemotherapy. ${ }^{35-37}$ As cIAP2 governs prosurvival responses to $\mathrm{TNF} \alpha$ and regulates innate immunity pathways, we hypothesized that it is a key regulator of intestinal homeostasis. Here we demonstrate that CIAP2 is a potent promoter of colitisassociated colorectal cancer (CAC) but is also a critical regulator of intestinal tissue repair following injury. Whereas ablation of cIAP2 decreased tumorigenesis, it was deleterious in the gut as it led to severe colitis and colitis-associated lethality. Mechanistically, cIAP2 exerted a dual function in the intestine, suppressing cell death while promoting interleukin-18 (IL-18)mediated intestinal epithelial cell (IEC) proliferation and tissue regeneration.

\section{RESULTS \\ Birc3 $^{-1-}$ mice are resistant to AOM-DSS-induced colitis- associated colorectal cancer}

Because of the prominent expression of cIAP2 in CRC and its role in innate immunity and cell survival, we sought to determine the response of cIAP2-deficient $\left(\mathrm{Birc}^{-/-}\right)$mice to CAC. Toward this aim, we used an established experimental animal model of CAC in which tumorigenesis is initiated with a single dose of the procarcinogen azoxymethane (AOM) and promoted by three cycles of $2 \%$ dextran sulfate sodium (DSS) in the drinking water that triggers intestinal injury and colitis (Figure 1a). Wild-type (WT) and Birc3 $3^{-1-}$ mice subjected to this treatment were killed on day 89 after AOM and examined for colonic tumor growth. At this concentration of DSS, there was no significant difference in the body weight of mice from the two genotypes (Supplementary Figure S1A online). However, tumorigenesis was markedly inhibited in the absence of cIAP2 (Figure 1b,c). Furthermore, the adenomas arising in Birc $3^{-/-}$ mice were markedly smaller in size compared with those in WT mice (Figure 1d,e), pointing to an important role of cIAP2 in tumor promotion. We next set to determine whether cIAP2 functioned in the stromal or hematopoietic compartment to mediate CAC. We generated bone marrow chimeras and investigated their response to AOM-DSS treatment. As expected, control Birc3 ${ }^{-/-}$mice receiving $\mathrm{Birc}^{-/-}$bone marrow $\left(\right.$ Birc $^{-/-}>\mathrm{Birc}^{-1^{-}}$) had fewer tumors than control WT mice transplanted with WT bone marrow (WT $>$ WT). On the other hand, Birc3 $3^{-1-}$ mice receiving WT bone marrow $\left(\mathrm{WT}>\mathrm{Birc}^{-1-}\right)$ phenocopied Birc $3^{-1-}$ mice, whereas WT recipients of cIAP2-deficient bone marrow $\left(\right.$ Birc $\left.^{-1-}>\mathrm{WT}\right)$ were similar to WT mice (Figure 1f). This indicated that cIAP2 deficiency in the radioresistant compartment resulted in decreased CAC. The resistance of $\mathrm{Birc}^{-/-}$mice to tumorigenesis did not correlate with differential proliferation 

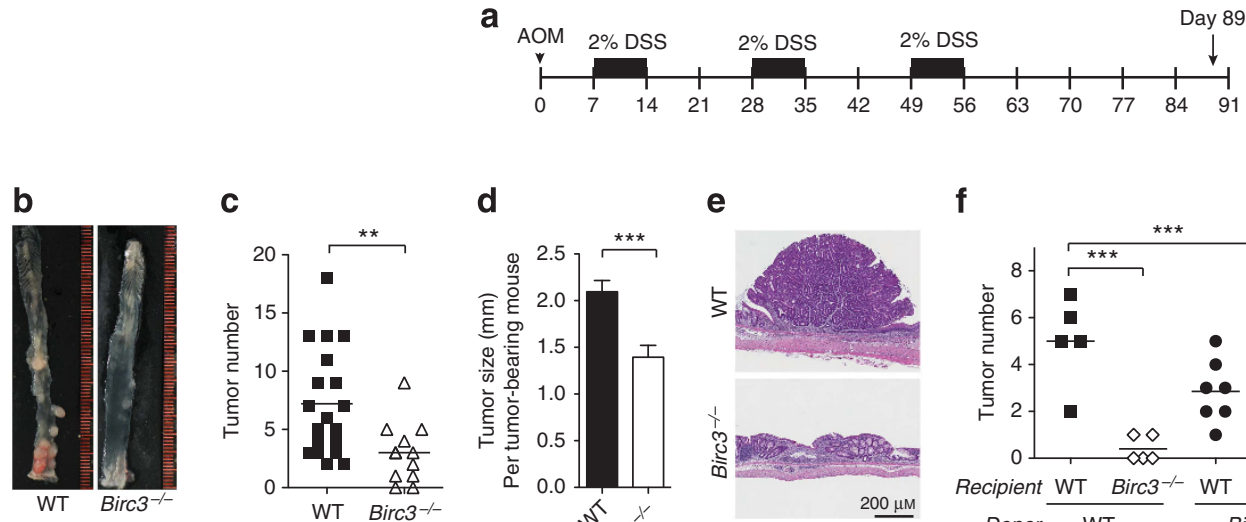

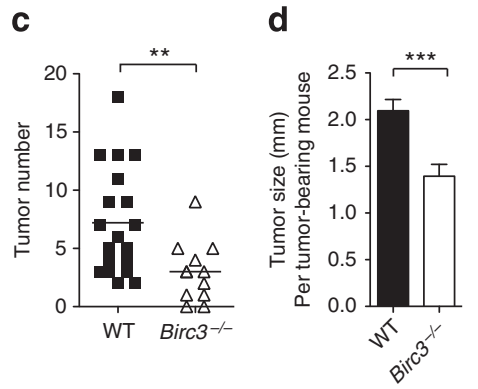

e

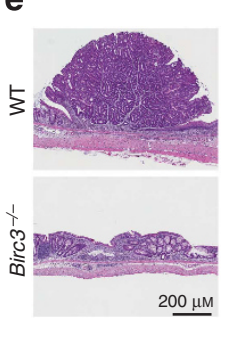

f

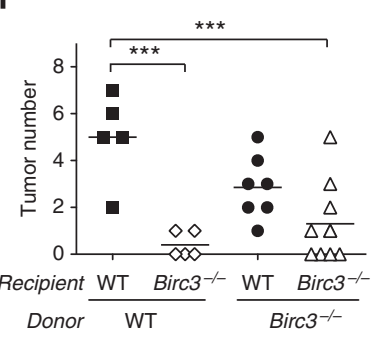

k

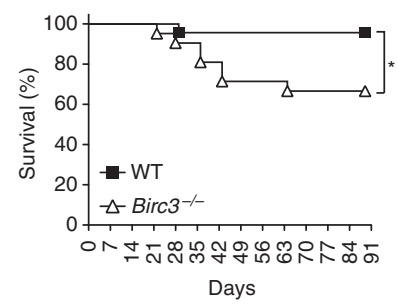

j

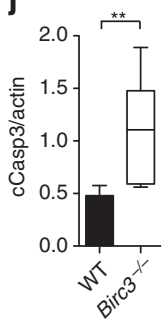

Figure $1 \mathrm{Birc} 3^{-/-}$mice are resistant to azoxymethane-dextran sulfate sodium (AOM-DSS)-induced colitis-associated colorectal cancer. (a) Schematic representation of the experimental procedure for the AOM-DSS model. Mice were injected with AOM $\left(10 \mathrm{mg} \mathrm{kg}^{-1}\right)$ on day 0 and allowed to recover for 1 week. On day 7,2\% DSS was given in the drinking water (black boxes) for 3 cycles of 7 days separated by 2 weeks of regular water. Mice were killed on day 89. (b) Representative macroscopic images of the colon from AOM-DSS-treated wild-type (WT) or Birc $3^{-1-}$ mice. (c) Numbers of colonic tumors in WT $(n=19)$ or Birc3 ${ }^{-1-}(n=12)$ mice following AOM-DSS treatment. Each symbol on the graph represents one mouse; the horizontal line represents the mean. Statistical analysis was performed using Student's $t$-test $(P=0.007)$. The data are pooled from two experiments. (d) Average tumor size of WT or Birc $3^{-1-}$ mice per tumor-bearing mouse. Data represent the mean \pm s.e.m. Statistical analysis was performed using Student's $t$-test $(P=0.0021)(\mathbf{e})$ Representative hematoxylin and eosin (H\&E) staining of colon sections from tumor-bearing WT and Birc $3^{-/-}$mice at experimental end point (day 89). (f) Tumor incidence induced by AOM-DSS in WT or Birc3 ${ }^{-1-}$ chimeric mice. Each symbol on the graph represents one mouse; the horizontal line represents the mean. Statistical analysis was performed using one-way analysis of variance (ANOVA; $n=5-10$ mice per group; $P<0.001$ ). (g) Epifluorescence images of tumors from colon sections from WT or Birc $3^{-/-}$mice on day 89 after AOM-DSS treatment stained with antibodies against proliferating cellular nuclear antigen (PCNA) to mark dividing cells, E-cadherin to mark intestinal epithelial cells (IECs), and Hoechst to label nuclei. Insets correspond to boxed regions. (h) Numbers of PCNA-positive cells per $\mathrm{mm}^{2}$ of tumor area. Data represent the mean \pm s.e. $\mathrm{m}$. Statistical analysis was performed using Student's $t$-test $(P>0.05)$. (i) Western blots depicting active caspase-3 and actin expression in colon homogenates from WT or Birc $3^{-\prime}$ mice following AOM-DSS treatment (day 89). Each lane represents one mouse. (j) Scatter plot of the densitometric ratio of cleaved caspase-3 (cCasp3) over actin. Statistical analysis was performed using Student's $t$-test $(P=0.0079)$. (k) Kaplan-Meier survival curves of WT $(n=20)$ and Birc3 $3^{-1-}(n=21)$ mice following AOM-DSS treatment. Statistical significance was calculated using Mantel-Cox test $(P=0.0263)$. The data are pooled from two experiments. The AOM-DSS experiments were repeated three times. The bone marrow chimera experiment was done once.

of cancer cells at end point as revealed by immunofluorescence staining using antibodies against the proliferation marker proliferating cellular nuclear antigen (PCNA) (Figure 1g,h). In contrast, enhanced activation of caspase- 3 was observed in colon homogenates from Birc $3^{-1-}$ mice compared with WT mice (Figure 1i $\mathbf{i} \mathbf{j})$, suggesting that expression of cIAP2 is necessary to curb apoptosis in CAC. Immunofluorescence staining of colon sections with TUNEL (terminal deoxynucleotidyl transferase dUTP nick end labeling), active caspase-3 antibodies, and E-cadherin labeling indicated that apoptosis occurred primarily in IECs (Supplementary Figure S1B). As Casp11 is in close proximity to Birc3 and carries an inactivating mutation in the 129 strain of mice, embryonic stem cells of which were used to generate Birc $3^{-1-}$ mice, ${ }^{38,39}$ we next verified the status of caspase-11 expression. Genotyping for the passenger mutation in Casp11 confirmed that our Birc3 ${ }^{-/-}$ mice were sufficient for caspase-11, as previously reported 22,40
(Supplementary Figure S1C). Immunoblot analysis further revealed that caspase- 11 was similarly expressed in the colon of WT and Birc3 ${ }^{-/-}$mice following AOM-DSS treatment (Supplementary Figure S1D), indicating that the phenotype observed in Birc3 $3^{-1-}$ mice was independent of alterations in caspase-11 levels. Similarly, the suppressed tumorigenesis phenotype of Birc3 $^{-1-}$ mice was not linked to differential activation of the canonical or noncanonical NF- $\kappa B$ pathways as levels of $\mathrm{I} \kappa \mathrm{B} \alpha$, processing of NF- $\mathrm{B} 2$ p100 into p52, and phosphorylation of the NF- $\mathrm{kB}$ p65 subunit were similar between genotypes (Supplementary Figure S1E-G). Altogether, our data show reduced tumorigenesis in $B i r c 3^{-/-}$ mice compared with WT mice that was independent of differences in NF- $\mathrm{KB}$ activation or cellular proliferation. However, apoptosis was more pronounced in $B i r c 3^{-/-}$mice, which might partially account for the decreased tumor burden in these mice. Interestingly, although $B i r c 3^{-1-}$ mice were more 
resistant to CAC than WT animals, they exhibited increased mortality to AOM-DSS, with $>45 \%$ of $\mathrm{Birc}^{-/-}$mice succumbing throughout the treatment (Figure 1k). We thus hypothesized that although cIAP2 expression promotes cancer, it might also be required for maintaining intestinal homeostasis and countering colitis.

\section{cIAP2 deficiency confers enhanced susceptibility to DSS- induced colitis}

The increased mortality of Birc3 $3^{-/-}$mice in response to AOMDSS treatment suggested that cIAP2 might be critical for mucosal healing following intestinal injury. In order to investigate this hypothesis, we subjected WT and Birc3 ${ }^{-/-}$ mice to models of acute or chronic DSS-induced tissue injury and colitis. For the acute model, 3\% DSS in the drinking water was administered for 5 days, followed by 3 days of regular drinking water, whereas this DSS cycle was administered twice separated by 10 days of rest for the chronic model (Figure 2a). Birc3 ${ }^{-/-}$ mice were significantly more susceptible than WT mice to both acute and chronic DSS treatment, as evidenced by severe shrinking of the colon at necropsy on days 8 and 23, an indicator of increased disease severity (Figure 2b,c). Consistently, Birc3 $^{-/-}$mice showed increased tissue injury, with $>70 \%$ or $>80 \%$ of the colon being damaged or fully eroded on days 8 and 23, respectively, compared with $60 \%$ in WT mice on either time point (Figure 2d,e). This differential response to DSS was not associated with marked differences in inflammatory infiltrates in the colon (Supplementary Figure S2A) and was independent of inherent differences between the two genotypes at baseline, as colon length and crypt architecture were similar between WT and Birc $3^{-/-}$mice (Supplementary Figure S2 B-D). To assess the kinetics of colitis induction and progression in the two genotypes, we next performed a timecourse experiment. Colon length measurements and histological analysis of crypt architecture indicated enhanced colitis in Birc $^{-/-}$mice as early as day 3 or 5 after DSS (Supplementary Figure S2 E-G). Next, we wished to explore whether, akin to the AOM-DSS model, cIAP2 mediated its response to DSS from the radioresistant compartment. We thus subjected bone marrow chimeras to $3 \%$ acute DSS treatment and measured survival as a readout of colitis severity. Mice lacking cIAP2 in the radioresistant compartment (WT $>$ Birc $3^{-/-}$) succumbed to DSS treatment similar to $B \operatorname{irc} 3^{-/-}>\mathrm{Birc}^{-/-}$mice, whereas Birc $^{-1-}>$ WT were as resistant as WT $>$ WT control mice (Supplementary Figure S2H), pointing to a key role of stromal expression of cIAP2 in intestinal homeostasis. As mentioned previously, IAP antagonists, or SMs, are being currently investigated in clinical trials as potential therapeutics for cancer. ${ }^{41}$ Monovalent SMs deplete both cIAP1 and cIAP2 with similar kinetics but are less efficient at depleting XIAP. ${ }^{42}$ To explore the impact of these compounds on intestinal homeostasis and response to injury independently of genetic deletion, we orally administered either DMSO vehicle control or one such SM, LCL161, to WT littermates treated with DSS using a regimen of $50 \mathrm{mg} \mathrm{kg}^{-1}$ every 3 days (Figure 2f). We monitored the expression of cIAP1 and XIAP as a readout for compound activity. cIAP1 was more efficiently depleted from the colon than $\mathrm{XIAP}$ as revealed by immunoblot at end point (Figure 2g). As for Birc3 $^{-/-}$mice, LCL-161-treated WT mice were significantly more susceptible to DSS-induced injury compared with mice treated with vehicle control, as evidenced by colon shortening (Figure 2h,i) and mucosal erosion (Figure 2j,k). Together, these results indicated that expression of cIAP2 in the colon is required for protection against DSS-induced injury and that depletion of the cIAPs by SM leads to intestinal tissue destruction and pathology.

Deficit in IEC compensatory proliferation after tissue injury mediated by impaired IL-18 production in Birc $^{-/-}$mice

To explore the mechanistic basis of enhanced disease severity in Birc3 ${ }^{-/-}$mice, we next set to determine whether differences in IEC compensatory proliferation were evident between the two genotypes and were at the basis of crypt erosion in Birc $3^{-1-}$ mice. To label proliferating IECs, we used immunohistochemistry to costain colon sections with antibodies against PCNA and the epithelial cell marker E-cadherin. There was no noticeable difference in the number of proliferating cells between WT and Birc $3^{-/-}$mice at baseline and the DSS-induced decrease in the number of these cells on day 3 or 5 after DSS was similar between genotypes (Supplementary Figure S3 A, B). However, there was a notable increase in IEC compensatory proliferation in WT mice on day 8 after DSS, but this response was markedly blunted in $B i r c 3^{-/}$mice (Figure 3a), suggesting a role for cIAP2 in crypt regeneration. Multiple cytokines, including IL-6, IL-11, IL-22, and IL-18, have been reported to mediate IEC proliferation and tissue repair following DSS injury. ${ }^{43}$ To characterize the inflammatory cytokines produced in the intestine of WT and Birc3 ${ }^{-/-}$mice after DSS, we determined by quantitative real-time PCR (qPCR) their expression levels on day 8 or 23 after DSS. All cytokines measured were similar at the mRNA level between the two genotypes (Figure 3c). We next cultured a small piece of the colon overnight and measured cytokine production in the organ culture supernatant by enzyme-linked immunosorbent assay. Whereas the production of IL-6, IL-11, and IL-1 $\beta$ was not impaired in Birc $3^{-/-}$mice compared with WT mice (Figure 3d), the marked induction of IL-18 observed in the colon of WT mice on day 8 or 23 after DSS was significantly attenuated in Birc $3^{-1-}$ mice (Figure 3d). Interestingly, in the early phase of the disease, IL-18, caspase-1, and caspase-11 levels, although slightly higher than homeostatic levels (day 0), were similar in the two genotypes (Supplementary Figure S3 C,D), correlating with lack of differences in IEC compensatory proliferation at these early time points (Supplementary Figure S3 B). We and others have previously demonstrated that IL-18 processing by caspase1 is required to initiate tissue repair following DSS injury. ${ }^{44-49}$ Furthermore, we have shown that CIAP1 and cIAP2 are required for efficient activation of caspase- $1 .^{29}$ The blunted production of IL-18 in Birc $3^{-/-}$mice at the end point of acute or chronic colitis was not observed at the mRNA level (Figure 3c), and was not caused by impaired Nlrp3 or Nlrp6 inflammasome priming, as components of these inflammasomes were equivalently 
a
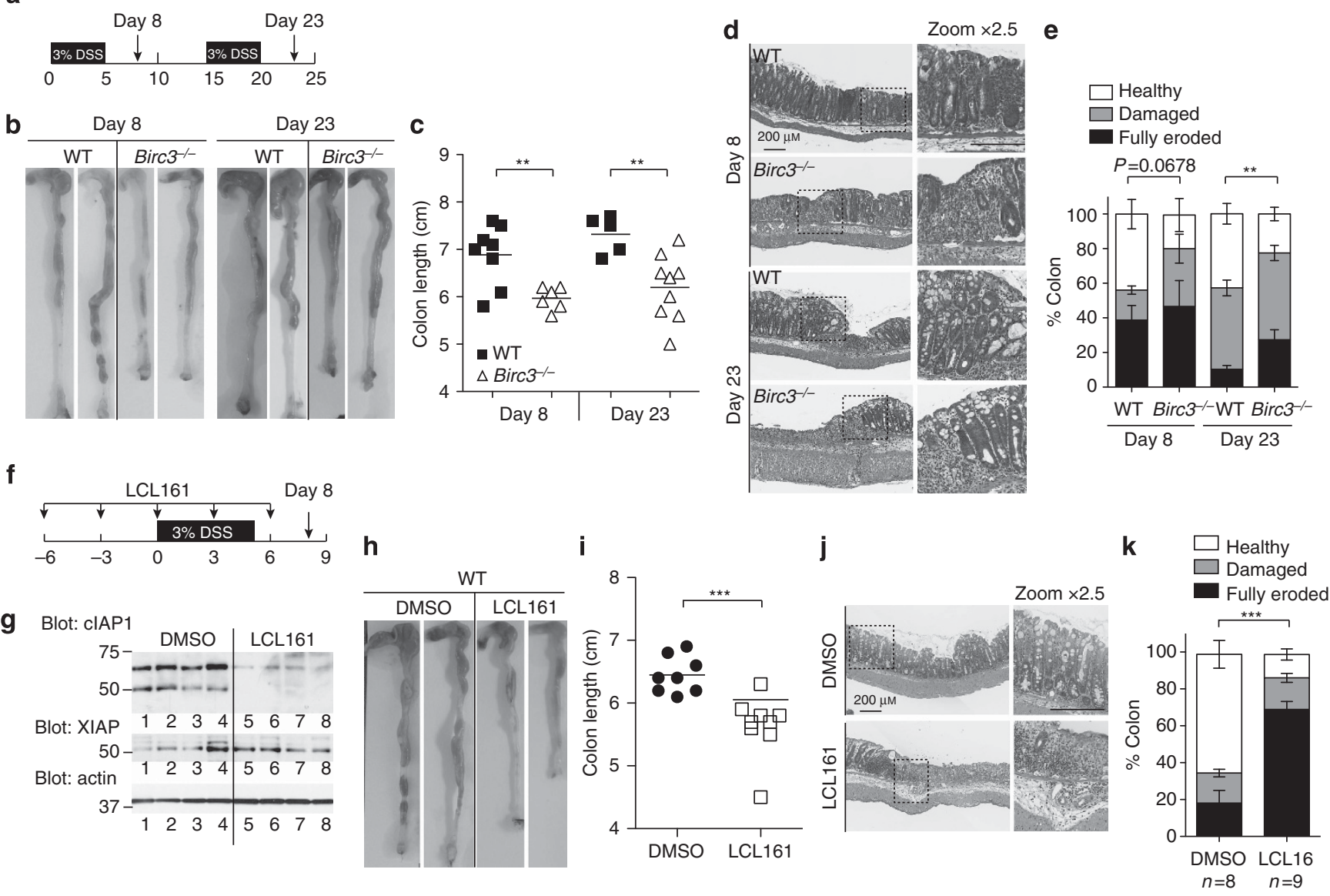

Figure 2 Loss of cellular inhibitor of apoptosis protein 2 (CIAP2) expression in mice leads to heightened susceptibility to dextran sulfate sodium (DSS)induced colitis. (a) Schematic representation of the experimental procedure for the DSS model. Mice were given $3 \%$ DSS in the drinking water for 5 days (black box), followed by 10 days of clean water. The cycle was repeated a second time in some experiments, as indicated. Mice were killed on days 8 or 23 (black arrows). (b) Representative photographs of colon and cecum from wild-type (WT) or Birc3 ${ }^{-/-}$mice on days 8 or 23 following DSS treatment. (c) Colon length of WT or Birc3 ${ }^{-1-}$ mice following DSS treatment on day 8 or day 23. Each symbol on the graph represents 1 mouse; (day 8: WT ( $n=8$ ), Birc3 $^{-1-}(n=6)$; day 23: WT $(n=5)$, Birc $\left.3^{-1-}(n=9)\right)$ the horizontal line represents the mean. Statistical analysis was performed using Student's $t$-test (day 8, $P=0.0060$; day 23, $P=0.0063$ ). (d) Representative hematoxylin and eosin (H\&E) staining of colon sections from WT or Birc3 ${ }^{-/-}$mice on days 8 or 23 following DSS treatment. Scale bar $=200 \mu \mathrm{m}$. Insets correspond to boxed regions. (e) Intestinal tissue damage and erosion were quantified as described in the Methods. Data represent the mean \pm s.e.m. Statistical analysis was performed using two-way analysis of variance (ANOVA; day 8 , $P=0.0678$; day 23, $P=0.0015$ ). (f) Schematic representation of the experimental procedure. WT male mice were orally gavaged with $50 \mathrm{mg} \mathrm{kg}^{-1}$ of LCL161 or vehicle control every 3 days starting on day -6 of the experiment (black arrows). On day 0 , mice were treated with $3 \%$ DSS for 5 days (black box), followed by 3 days of clean water. The experiment ended on day 8 (dimethyl sulfoxide (DMSO), $n=8$; LCL161, $n=9$ ). (g) Western blots depicting CIAP1, XIAP (X-linked inhibitor of apoptosis protein), and actin levels in colon homogenates from WT mice treated with LCL161 or DMSO on day 8 following DSS treatment. Each lane represents one mouse. (h) Representative photographs of colon and cecum from WT mice gavaged with DMSO or LCL161 on day 8 following DSS treatment. (i) The colon length of LCL161- or vehicle-treated WT mice following DSS treatment is shown (day 8 ). Each symbol on the graph represents one mouse; the horizontal line represents the mean. Statistical analysis was performed using Student's $t$-test $(P=0.0010)$. (j) Representative H\&E staining of colon sections derived from mice on day 8 after the start of DSS treatment. Scale bar $=200 \mu \mathrm{m}$. Insets corresponds to boxed regions. (k) Intestinal tissue damage and erosion were quantified as described in the Methods. Data represent the mean \pm s.e.m. Statistical analysis was performed using two-way ANOVA $(P<0.0001)$. Day 8 end point experiments were repeated twice and day 23 end point experiments were repeated four times with similar results. LCL161 data are representative of two separate experiments.

expressed in WT and Birc3 ${ }^{-1-}$ mice at both the mRNA and protein levels (Figure 3f and Supplementary Figure S3E). Consistently, there were no differences in the extent of activation of the canonical or noncanonical NF- $\kappa B$ pathways, extracellularsignal-regulated kinase, or p38 mitogen-activated protein kinase in the colon of DSS-treated WT or Birc $3^{-/-}$mice as determined by immunoblot (Supplementary Figure S3F). These results point to a requirement for cIAP2 in promoting caspase-1 activation in vivo in response to DSS injury. Consistently, immunoblot analysis showed increased caspase- 1 activation in the colon homogenates of WT mice compared with $\mathrm{Birc}^{-1-}$ animals on both days 8 and 23 after DSS (Figure 3e). Caspase-11, a caspase-1-related inflammatory caspase, was recently reported to play a critical role in intestinal homeostasis but its mechanism of action is debated. In one report, caspase- 11 was suggested to act independently of IL- $1 \beta$ and IL- $18,{ }^{50}$ whereas it was shown to regulate intestinal IL-18 levels in another report. ${ }^{51}$ Examination of caspase-11 expression following DSS treatment revealed no differences in the colon homogenates of WT or Birc3 ${ }^{-/-}$ animals (Supplementary Figure S3G), reinforcing that the role 


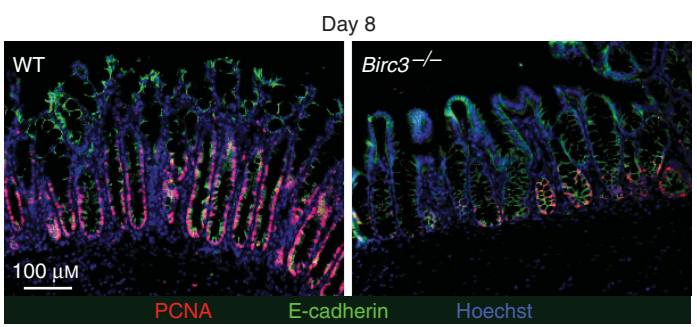

b

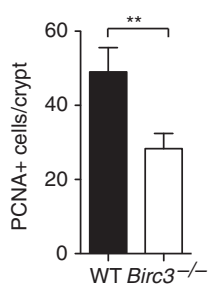

C

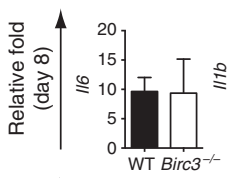

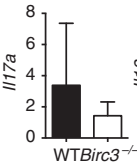
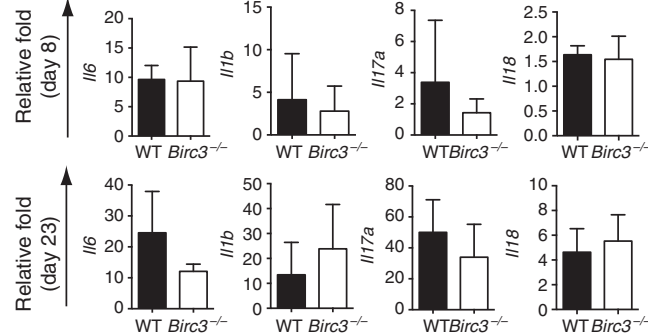

10

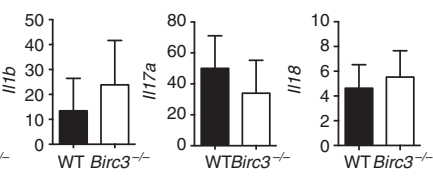

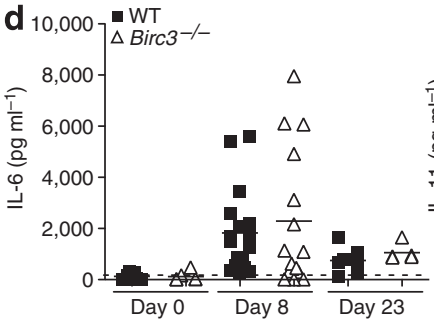
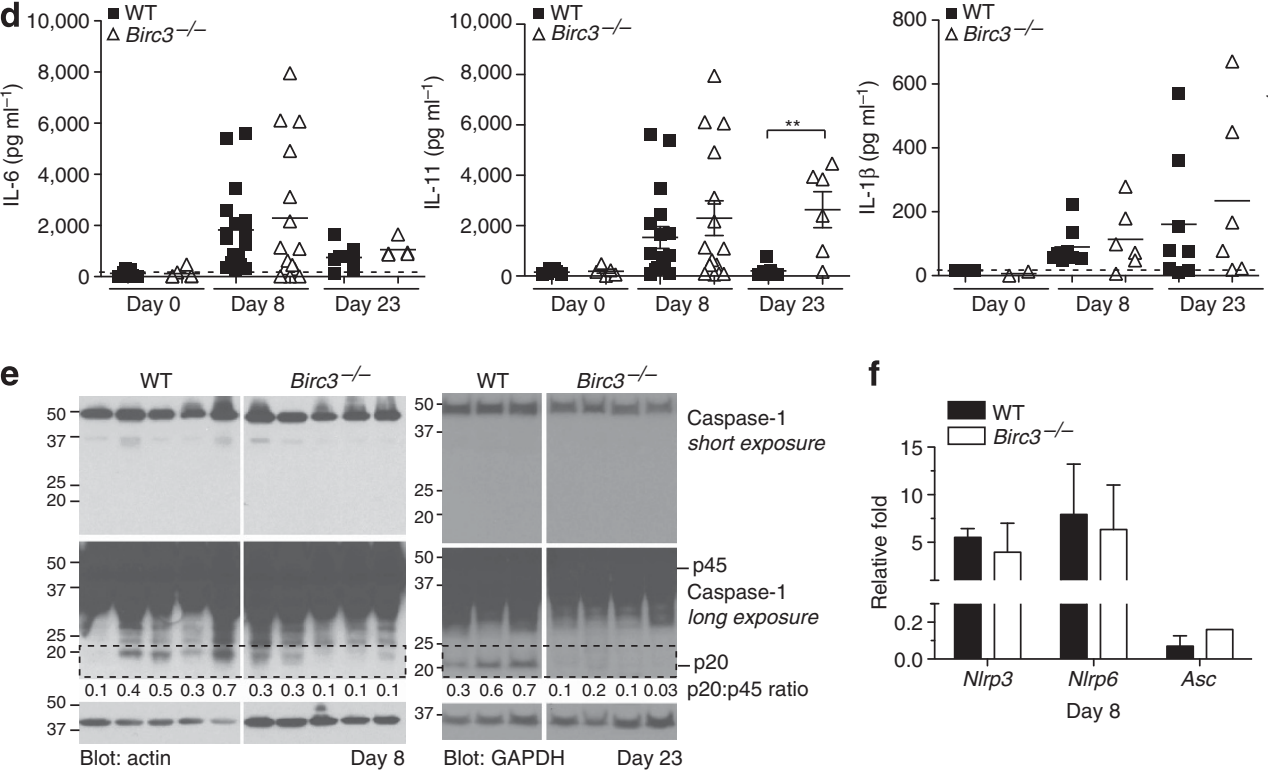

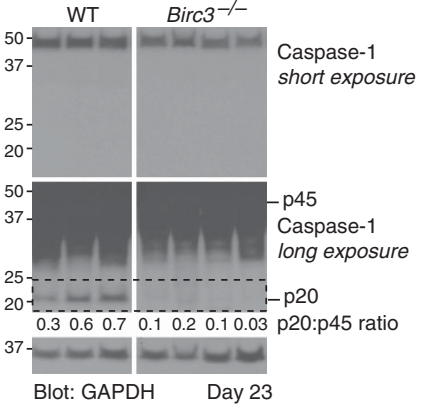

f

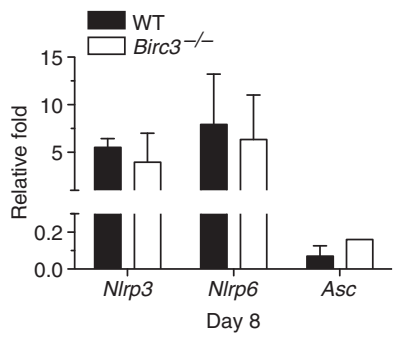

g

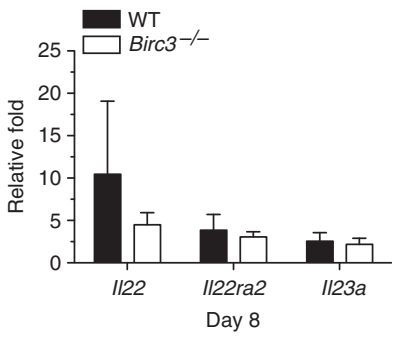

Figure 3 Impaired intestinal epithelial cell compensatory proliferation and interleukin-18 (IL-18) production following dextran sulfate sodium (DSS) in Birc $3^{-1-}$ mice. (a) Epifluorescence images of colon sections from wild-type (WT) or Birc ${ }^{-1-}$ mice on day 8 after DSS treatment stained with antibodies against proliferating cellular nuclear antigen (PCNA) to mark dividing cells, E-cadherin to mark intestinal epithelial cells (IECs), and Hoechst to label nuclei. (b) The numbers of PCNA-positive cells per crypt is shown (7-12 crypts were scored per mouse; 3 mice per genotype were quantified). Data represent the mean \pm s.e.m. Statistical analysis was performed using Student's $t$-test $(P=0.0085)$. (c) mRNA derived from the colon of WT or Birc3 ${ }^{-\prime}$ mice on day 8 or 23 after DSS treatment was analyzed for expression of $/ / 6,\|/ 1 b,\| / 17 a$, and $/ / 18$ by quantitative real-time PCR (qPCR). Data represent the mean \pm s.d. Statistical analysis was performed using Student's $t$-test $(n=6-8$ mice per genotype; $P>0.05)$. (d) Colons from WT or Birc $3^{-\prime-}$ mice were dissected on days 0,8 , or 23 after DSS treatment. After flushing and thoroughly washing the colon, $0.5 \mathrm{~cm}$ pieces were cultured overnight to assess cytokine production. Enzyme-linked immunosorbent assays (ELISAs) for IL-6, IL-11, IL-1 $\beta$, and IL-18 were performed on supernatants of organ culture. Each symbol on the graph represents one mouse; the horizontal line represents the mean. The horizontal dotted line represents the lower detection limit of the assay. Statistical analysis was performed using Student's $t$-test $(n=5-9$ mice after DSS per time point per genotype, IL-18: day 8, $P=0.0077$, day 23, $P=0.0007$ ). (e) Western blots depicting caspase-1 and actin (day 8) or GAPDH (day 23) levels in colon homogenates from DSS-treated WT or Birc ${ }^{-}{ }^{-1}$ mice. Densitometry ratio analysis of caspase-1 p20 over p45 is shown below. (f) mRNA derived from the colon of WT or Birc $3^{-1-}$ mice on day 8 after DSS treatment was analyzed for expression of NIrp3, NIrp6, and Asc by qPCR. Data represent the mean \pm s.d. Statistical analysis was performed using Student's $t$-test ( $n=6-8$ mice per genotype). (g) mRNA derived from the colon of WT or Birc ${ }^{-1-}$ mice on day 8 after DSS treatment was analyzed for expression of II22, II22ra2, and II23a by qPCR. Data represent the mean \pm s.d. Statistical analysis was performed using Student's $t$-test $(n=6-8$ mice per genotype). Day 8 end point experiments were repeated twice and day 23 end point experiments were repeated four times.

of cIAP2 in regulating IL-18 production and intestinal tissue repair is independent of this protease. IL-18 was shown to promote tissue repair partially through regulation of the stoichiometry of IL-22/IL-22-binding protein (BP). ${ }^{43}$ To examine whether cIAP2 modulated this axis, we measured levels of IL22 and IL-22BP in the colon of Birc $3^{-1-}$ mice compared with WT animals on day 8 following DSS treatment. A trend toward reduced IL-22 mRNA was observed in the colon of Birc $3^{-/-}$ mice compared with WT mice but this difference did not attain statistical significance (Figure 3g). In contrast, no differences were seen for IL-22BP (Il22ra2) or IL-23 (Figure 3g). Together, these data suggest that cIAP2 is required for IL-18 production that is needed for adequate intestinal epithelial cell proliferation and tissue regeneration following damage. Notably, compared with $\mathrm{Ice}^{-/-}$or $\mathrm{Ill8^{-/- }}$ mice that succumb readily after one cycle of DSS, ${ }^{44,45,49}$ Birc $^{-1-}$ mice have an intermediate colitis phenotype, surviving through for over two DSS cycles. We thus wished to determine whether residual IL-18 production in Birc3 ${ }^{-/-}$mice further contributed to IEC proliferation and tissue repair. We addressed this question by administering AOM-DSS-treated $\mathrm{Birc}^{-1-}$ mice daily injections of either phosphate-buffered saline (PBS) or the IL-18 inhibitory protein IL-18BP (Supplementary Figure S3H). Interestingly, IL-18BP markedly blunted the residual IEC proliferation in 

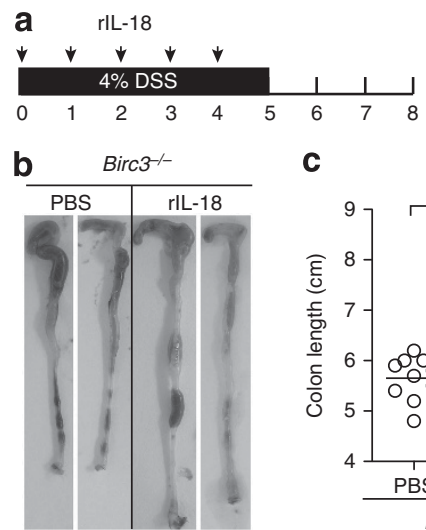

C

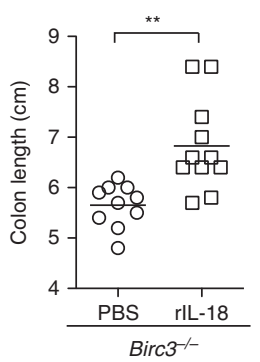

f

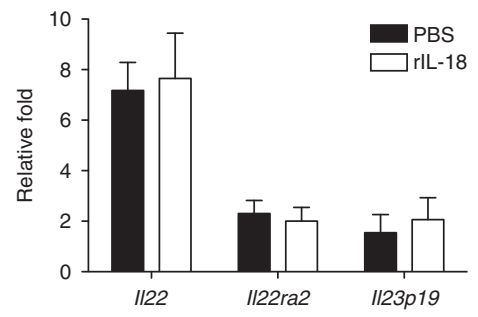

e
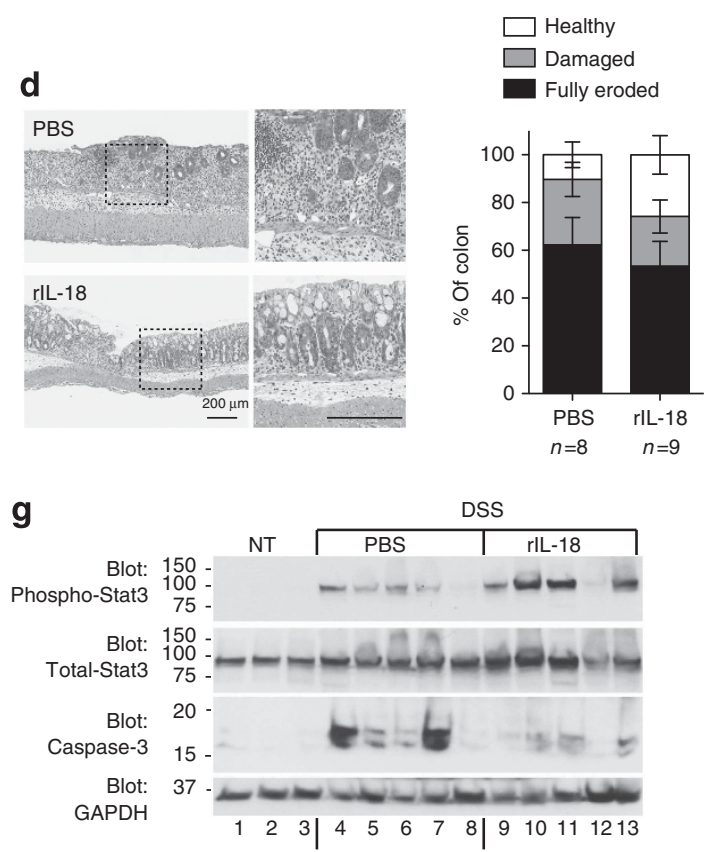

Figure 4 Murine recombinant interleukin-18 (IL-18) treatment of Birc $3^{-/-}$mice alleviates their severe dextran sulfate sodium (DSS)-induced colitis phenotype. (a) Schematic representation of the experimental procedure for the DSS model. Birc3 ${ }^{-1-}$ mice were treated with $4 \%$ DSS for 5 days, followed by 3 days of clean water. Mice were injected daily from days 0 to 4 with either phosphate-buffered saline (PBS) or recombinant IL-18 (rlL-18; $0.05 \mu \mathrm{g})$. (b) Representative photographs of colon and cecum from Birc3 ${ }^{-1-}$ mice injected with PBS or rlL-18 on day 8 following DSS treatment. (c) Colon length of DSS-treated mice is shown. Each symbol on the graph represents one mouse; the horizontal line represents the mean. Statistical analysis was performed using Student's $t$-test $(P=0.0014)$. Data are representative of three separate experiments with similar results. (d) Representative hematoxylin and eosin (H\&E) staining of colon sections from Birc $3^{-1-}$ mice on days 8 . Scale bar $=200 \mu \mathrm{m}$. Insets correspond to boxed regions. (e) Intestinal tissue damage and erosion were quantified as described in the Methods. Data represent the mean \pm s.e.m. Statistical analysis was performed using two-way analysis of variance (ANOVA). The data are representative of three separate experiments. (f) mRNA derived from the colon of Birc3 $3^{-1-}$ mice injection with rIL-18 or PBS on day 8 after DSS treatment was analyzed for expression of II22, II22ra2, and II23a by quantitative real-time PCR (qPCR). Data represent the mean \pm s.d. Statistical analysis was performed using Student's $t$-test ( $n=6-7$ mice per time point). (g) Western blots depicting phospho-STAT3, total STAT3 (signal transducer and activator of transcription 3), cleaved caspase-3, and glyceraldehyde 3-phosphate dehydrogenase (GAPDH) levels in colon homogenates from untreated (NT) Birc3 ${ }^{-1-}$ mice or DSS-treated Birc3 ${ }^{-1-}$ mice injected with PBS or rlL-18. Each lane represents one mouse.

Birc3 $^{-1-}$ mice (Supplementary Figure S3 I,J), further confirming the key role of IL-18 in compensatory proliferation.

\section{IL-18 is sufficient to protect Birc3 ${ }^{-1-}$ mice from DSS-induced colitis.}

In order to assess whether the decreased IL-18 production was causative of the susceptibility phenotype observed in Birc3 ${ }^{-/-}$mice, we administered either PBS or exogenous murine recombinant IL-18 (rIL-18) to Birc $3^{-1-}$ littermate mice intraperitoneally at a regimen of $0.05 \mu \mathrm{g}$ per mouse daily from day 0 to day 4 after DSS (Figure 4a). We chose to use $4 \%$ DSS in this case to ensure induction of severe colitis and examine the impact of rIL-18 in a more aggressive model of acute tissue injury. Interestingly, exogenous administration of rIL-18 rescued the susceptibility phenotype of Birc3 ${ }^{-1-}$ mice. The colon of Birc3 ${ }^{-/-}$mice injected with PBS were significantly shorter (Figure $\mathbf{4 b}, \mathbf{c}$ ), and markedly more eroded (Figure 4d,e), than colons of Birc $3^{-1-}$ mice receiving rIL-18, in which the crypt architecture was conserved to a higher degree. On the other hand, rIL-18 treatment did not impact the response of WT mice to DSS (Supplementary Figure S4 A-D). To further interrogate whether IL-18 modulated the IL-22 repair pathway, we quantified expression levels of IL-22, IL-22BP, and IL-23 in the colon of treated mice by $\mathrm{qPCR}$. Figure $4 \mathrm{f}$ shows that these genes were induced to similar levels in the presence or absence of rIL-18 and their expression did not correlate with the reparative effects of rIL-18 in the colon following DSS. To explore the mechanisms induced by rIL-18, we examined by immunoblot analysis of colon homogenates the expression and/or activation of STAT3 (signal transducer and activator of transcription 3), NF- $\mathrm{kB}$, caspase-3, and RIP3. Whereas canonical or noncanonical NF- $\mathrm{KB}$ signaling, RIP3 levels, and induction of $I l 6$ or $I l 11$ expression were similar at end point in the colon of mice treated with PBS or rIL-18 (Supplementary Figure S4 E, F), rIL-18 administration resulted in enhanced STAT3 phosphorylation and decreased caspase-3 activation (Figure $\mathbf{4 g}$ ). Taken the established role of STAT3 in IEC survival and protection for DSS-induced tissue injury, ${ }^{52,53}$ our results suggest that cIAP2 promotes tissue repair via inflammasome activation and IL-18-mediated STAT3 activation. 
a
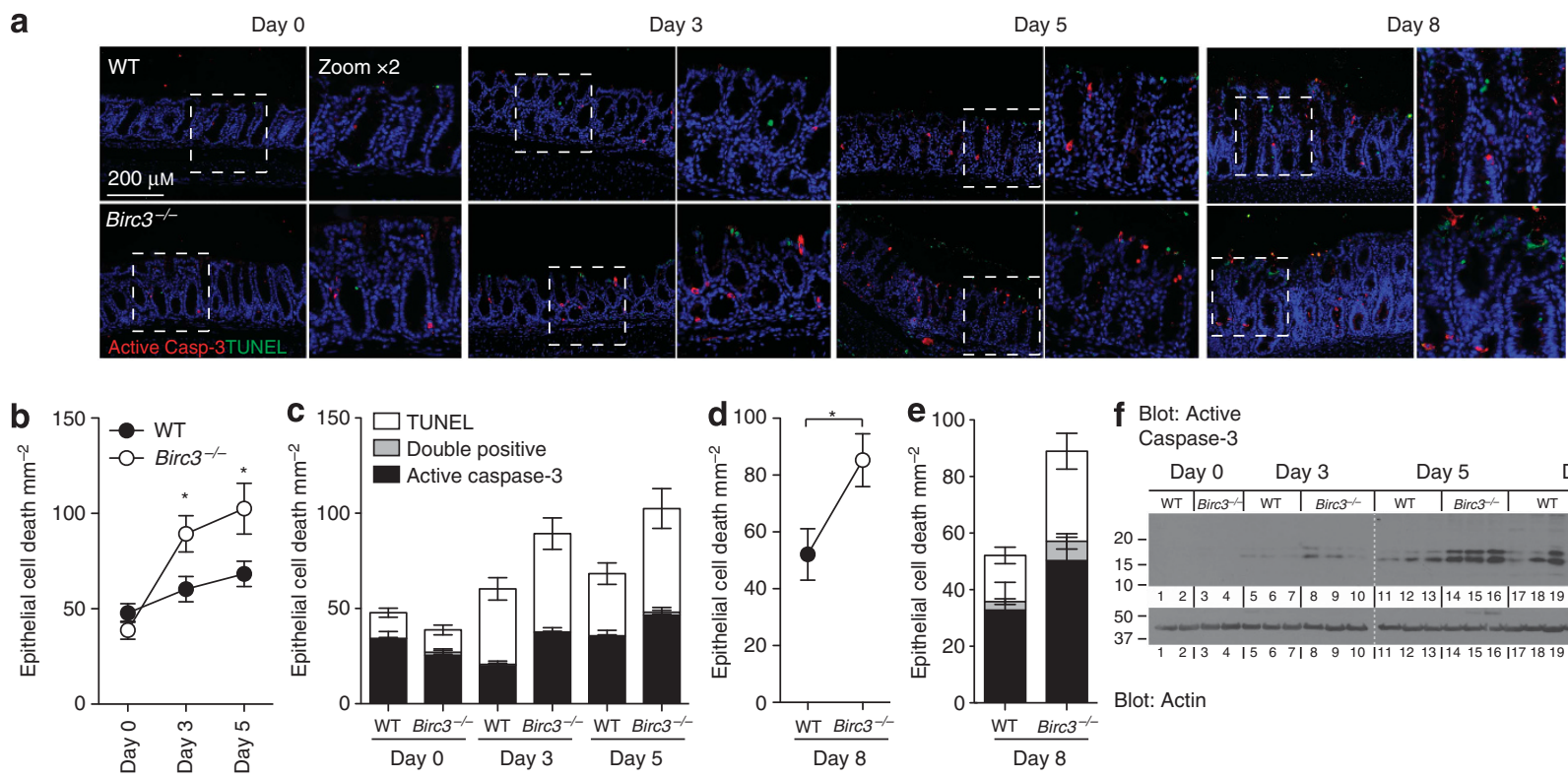

f Blot: Active

Caspase-3

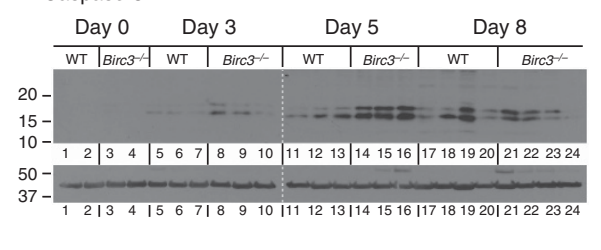

g

h

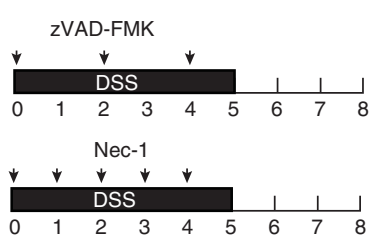

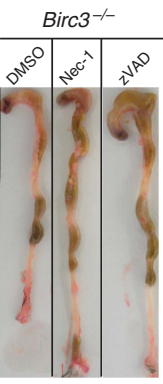

i

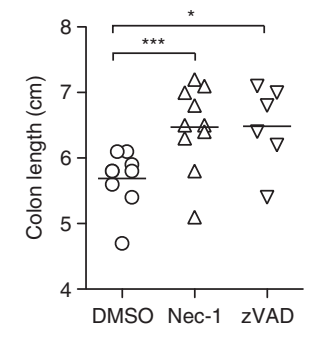

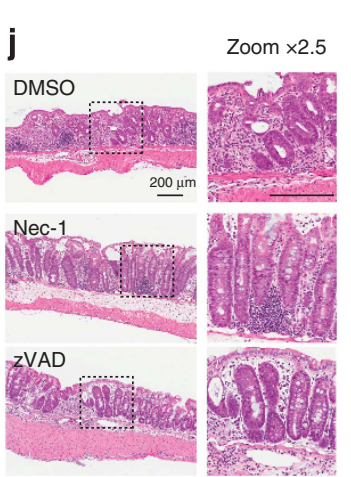

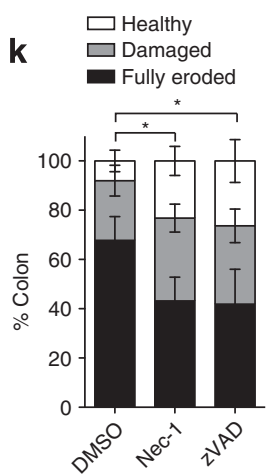

Figure 5 Blockade of RIP1 kinase activity or pan-caspase inhibition protects Birc3 ${ }^{-/}$mice from severe dextran sulfate sodium (DSS)-induced colitis. (a) Epifluorescence images of colon sections from wild-type (WT) or Birc $3^{-1-}$ mice on days $0,3,5$, and 8 after DSS treatment stained with antibodies against active caspase-3 and TUNEL (terminal deoxynucleotidyl transferase dUTP nick end labeling). Insets correspond to boxed regions. (b) Epithelial cell death (defined as cells positive for active caspase-3 and/or TUNEL staining) was quantified from 5-7 distinct colon sections per mouse (area $0.25 \mathrm{~mm}^{2}$ ), 3 mice per genotype. The data point represents the mean \pm s.e.m. Statistical analysis was performed using Student's $t$-test (day 0 , not significant (NS); day 3, $P=0.0207$; day 5, $P=0.026$ ). (c) Stratification of epithelial cell death as either caspase-3, TUNEL, or double positive on days 0 , 3 , and 5. Data represent the mean \pm s.e.m. (d) Epithelial cell death (defined as cells positive for active caspase-3 and/or TUNEL staining) was quantified from four distinct colon sections per mouse (area $0.25 \mathrm{~mm}^{2}$ ), three mice per genotype. The data point represents the mean \pm s.e.m. Statistical analysis was performed using Student's $t$-test $(P=0.0249)$. (e) Stratification of epithelial cell death as either caspase-3, TUNEL, or double positive. Data represent the mean \pm s.e.m. (f) Western blots depicting cleaved caspase-3 and actin levels in colon homogenates from WT or Birc $3^{-1-}$ mice on days $0,3,5$, and 8 after DSS treatment. Each lane represents one mouse. (g) Schematic representation of the experimental procedure for the DSS model. Birc ${ }^{-1-}$ mice were treated with $4 \%$ DSS for 5 days, followed by 3 days of clean water. Mice were injected daily from days 0 to 4 with either Necrostatin-1 (Nec-1; $\left.5 \mathrm{mg} \mathrm{kg}^{-1}\right)$ or vehicle control, or on days 0,2 , and 4 with zVAD-FMK $\left(3 \mathrm{mg} \mathrm{kg}^{-1}\right)$ or vehicle control $(n=6-9$ mice per treatment). (h) Representative photographs of colon and cecum from Birc $3^{-1}$ mice injected with vehicle control or inhibitor on day 8 following DSS treatment. (i) Colon length of DSStreated mice was measured on day 8 . Each symbol on the graph represents one mouse; the horizontal line represents the mean. Statistical analysis was performed using Student's $t$-test (Nec-1, $P=0.0063$; zVAD-FMK, $P=0.0123$ ) (j) Representative hematoxylin and eosin (H\&E) staining of colon sections from Birc $3^{-1-}$ mice on day 8. (k) Intestinal tissue damage and erosion were quantified as described in the Methods. Data represent the mean \pm s.e.m. Statistical analysis was performed using two-way analysis of variance (ANOVA; Nec-1, $P=0.0175 ; \mathrm{zVAD}-\mathrm{FMK}, P=0.0367$ ). The Nec-1 data are pooled from two experiments. The zVAD-FMK data are from one experiment.

\section{Inhibition of cell death by cIAP2 protects from DSS-induced injury}

In addition to regulating inflammation and innate immunity, the canonical function of the cIAPs is to antagonize apoptosis and necroptosis by preventing the formation of a ripoptosome downstream of death receptors or pattern recognition receptors such as Toll-like receptor-3 or $-4{ }^{54-56}$ To investigate the role of cell death in DSS-induced colitis, colon sections from WT or
Birc3 ${ }^{-1-}$ mice were labeled by immunofluorescence with active caspase- 3 antibodies and TUNEL. This analysis revealed the presence of both apoptosis- and caspase-3-independent cell death following DSS treatment in the two genotypes, but the overall number of epithelial dead cells in the colon was higher in Birc3 ${ }^{-1-}$ mice compared with WT mice on days 3, 5 , or 8 after DSS (Figure 5a-e). Analysis of caspase-3 activation kinetics revealed elevated caspase- 3 activation in Birc $3^{-1-}$ animals as 
early as day 3 or 5 after DSS (Figure 5f). In order to investigate which cell death modality was pathogenic in this model, we injected Birc3 $3^{-1-}$ mice intraperitoneally with the pan caspase inhibitor zVAD-FMK at a regimen of $3 \mathrm{mg} \mathrm{kg}^{-1}$ on days 0,2 , and 4 after DSS or with the RIP1 kinase inhibitor necrostatin-1 (Nec-1) at a regimen of $5 \mathrm{mg} \mathrm{kg}^{-1}$ daily from days 0 to 4 after DSS (Figure 5g). Interestingly, both inhibitors attenuated disease severity in Birc3 ${ }^{-/-}$mice, as revealed by improved colon anatomy (Figure $\mathbf{5 h , i}$ ) and crypt architecture (Figure $5 \mathbf{j}, \mathbf{k}$ ), but had no impact on WT mice (Supplementary Figure S4A-D). Of note, the kinase activity of RIP1 is not only required for necroptosis but also for apoptosis. ${ }^{57}$ Therefore, the rescue observed with $\mathrm{Nec}-1$ could be because of inhibition of apoptosis, necroptosis, or both. Immunoblot analysis of components of the apoptosis and necroptosis pathways revealed enhanced caspase- 8 activation on day 23 after DSS in colon homogenates from $\mathrm{Birc}^{-/-}$mice compared with WT mice in the presence of similar levels of the pronecrotic factors RIP3 and $\mathrm{cFLIP}_{\mathrm{S}}$ (Supplementary Figure S5A), suggesting a predominant role of apoptosis rather than other forms of cell death in mediating disease severity in $B i r c 3^{-1-}$ mice. Consistent with this model, both Ripk $3^{-1-}$ and Birc $3^{-/-} /$Ripk $^{-/-}$mice exhibited severe colitis pathology even more so than Birc $3^{-1-}$ mice in response to DSS. More than $75 \%$ of either Ripk $3^{-1-}$ or Birc3 ${ }^{-1-} /$ Ripk $^{-1-}$ mice succumbed to the DSS treatment compared with $34 \%$ Birc $^{-/-}$mice and 17\% WT mice, respectively (Supplementary Figure S5B). On day 8 after DSS, the colons of $\mathrm{Birc}^{-1-}$, Ripk $3^{-/-}$, and Birc $3^{-/-} /$Ripk $^{-/-}$mice were significantly shorter (Supplementary Figure S5C) and more eroded (Supplementary Figure S5D,E) than those of WT mice. Collectively, these results suggest that RIP3 exerts critical functions in the gut necessary for intestinal repair. The observation that single ablation of Ripk 3 led to severe disease independently of cIAP2 expression suggests additional functions of RIP3 in the gut besides necroptosis.

\section{DISCUSSION}

CRC is often linked to chronic inflammation arising from lesions at sites of tissue damage. Consistently, IBD patients have a higher risk of developing CAC. ${ }^{1}$ Experimentally, mouse strains that are susceptible to DSS-induced injury generally exhibit higher tumor burden after AOM-DSS treatment. It is posited that a deficit in barrier integrity following DSS allows microbial translocation and tissue damage-associated chronic inflammation that promotes tumorigenesis in the AOM-DSS model. Surprisingly, we found that Birc $3^{-/-}$mice are resistant to tumor formation following AOM-DSS, despite exhibiting elevated susceptibility to acute and chronic DSS-induced colitis, mediated by elevated cell death coupled with reduced caspase- 1 activation, IL-18 production, and IEC compensatory proliferation. Exogenous administration of recombinant IL-18 enhanced STAT3 phosphorylation and reversed the colitisassociated tissue damage in Birc3 ${ }^{-/-}$mice restoring crypt architecture. We did not detect differential induction of IL-6, IL-11 or IL-22 by rIL-18 in Birc3 ${ }^{-/-}$mice suggesting that either the impact of IL-18 on these cytokines is transient or that other mechanisms might converge on STAT3 activation. Interestingly, it was recently shown that suppression of SOCS3 expression by IL-1R signaling led to enhanced STAT3 activation in $\mathrm{T}$ cells. ${ }^{58}$ Whether this or other mechanisms are engaged downstream of IL-18 in the intestine requires further exploration. Both Nlrp3 and Nlrp6 have been previously implicated in DSS-induced caspase-1 activation and IL-18 production that was demonstrated to be crucial for crypt regeneration following injury. ${ }^{44-49}$ In the absence of inflammasome-induced repair, mice with targeted deletion of Casp1 or Il18, among others, were highly susceptible to AOM-DSS tumorigenesis. ${ }^{44,47,59}$ Our results demonstrating that IL-18BP administration to Birc3 ${ }^{-/-}$ mice abrogated any residual IEC proliferation following AOM injection and one cycle of DSS suggest that the attenuated production of IL-18 in Birc $3^{-1-}$ mice, although affecting tissue repair efficiency, might not be sufficiently damaging to promote CAC; however, this requires further investigation. More importantly, the added function of cIAP2 in cell death suppression might contribute to cancer promotion independently of damage-associated inflammation that implicates cIAP2 in nononcogenic tumor addiction. The increased susceptibility to DSS colitis yet enhanced resistance to AOM-DSS-induced CAC appears to be an "exception to the rule," but this phenotype is not confined to Birc $3^{-1-}$ mice. Indeed, IEC-specific ablation of NF- $\kappa \mathrm{B},{ }^{60}$ or STAT3 activation downstream of IL- $6^{52}$ or IL-1 $1{ }^{53}$ leads to a similar phenotype that is primarily mediated by impaired survival of normal and premalignant IECs.

Our results are consistent with previous work demonstrating enhanced CAC in mice deficient in SMAC that correlated with enhanced expression of cIAP2 ${ }^{61}$ It is interesting that in Birc3 $^{-/-}$mice, other IAPs, including cIAP1 and XIAP, do not compensate for the loss of cIAP2 in intestinal homeostasis in this model. As cIAP2 is highly inducible by inflammatory triggers, ${ }^{38}$ it might be particularly required to protect critical cell types from inflammation-associated cell death and tissue injury. XIAP contributes to intestinal homeostasis via an apparently distinct mechanism, specifically through mediating NOD1 and NOD2 signaling. ${ }^{62}$ Although inactivating mutations in human XIAP (BIRC4) cause the primary immunodeficiency X-linked lymphoproliferative disease type 2 (XLP2), a proportion of XLP2 patients exhibit intestinal inflammation. ${ }^{63-68}$ More recently, the link between XIAP and IBD was made more concrete with the identification of a number of $X I A P$ variants linked to early-onset Crohn's disease in $\sim 4 \%$ of males. ${ }^{69} \mathrm{In}$ all instances examined, these variants resulted in a defective NOD1/2 signaling and an impaired NF- $\kappa B$ response following NOD stimulation. ${ }^{69}$ However, whether cell death is involved in the intestinal pathology mediated by XIAP mutations remains to be elucidated.

Deregulation of the signaling balance downstream of TNF $\alpha$ leads to epithelial barrier breach and intestinal pathology. IECspecific targeted deletion of effectors of the NF- $\kappa B$ pathway, such as NEMO (NF- $\mathrm{KB}$ essential modulator) or both IKK $\alpha /$ IKK $\beta$, results in spontaneous colitis caused by excessive 
apoptosis. $^{70}$ Similarly, exacerbated IEC apoptosis leads to spontaneous intestinal inflammation and early lethality in mice with IEC-specific ablation of Ripk1., ${ }^{9,10}$ The premature death of these animals is partially rescued by Tnfrl deficiency; however, the contribution of the microbiota and Myd88 signaling to intestinal disease in this case is contested. Interestingly, the protective function of RIP1 in intestinal homeostasis is mediated by its scaffold function but appears to be independent of NF- $\kappa \mathrm{B}$, indicating that RIP1 directly suppresses caspase-8dependent apoptosis without de novo transcriptional induction of prosurvival factors by NF- $\kappa \mathrm{B}$. We show that the colitis phenotype of $B i r c 3^{-1-}$ mice is alleviated by administration of Nec-1, a pharmacological inhibitor of the RIP1 kinase, that is involved in both apoptosis and necroptosis. ${ }^{57}$ As caspase- 8 was robustly activated in the colon of DSS-treated $\mathrm{Birc}^{-1-}$ mice, in the presence of equivalent levels of the pronecrotic factors RIP3 and cFLIP $_{S}$, and taken the protective effects of zVAD-FMK, we propose that apoptosis rather than necroptosis is the primary pathological cell death in the colon of DSStreated mice. Concordantly, mice deficient in the proapoptotic protein PUMA were resistant to DSS colitis ${ }^{71}$ but highly susceptible to AOM-DSS-induced CAC, ${ }^{72}$ highlighting the critical role of apoptosis regulation in disease pathogenesis in this model. Interestingly, we found that Ripk3-deficient mice were highly susceptible to DSS colitis, irrespective of cIAP2 expression, suggesting extrinsic cell death-independent function of RIP3 in intestinal homeostasis that requires further investigation.

In summary, this study shows a critical role for cIAP2 in gut homeostasis. Following injury, cIAP2 is required to protect the IEC layer from tissue damage and to promote cell proliferation following injury. However, its expression in a $\mathrm{CRC}$ context leads to tumor promotion by maintaining cancer cell survival.

\section{METHODS}

Animal strains. WT, cIAP2 (Birc3-/- $)$-, Ripk3 (Ripk3 $\left.{ }^{-/-}\right)$-, and Ripk3/cIAP2 (Ripk3 $3^{-/-} /$Birc $^{-/-}$)-deficient mice on a C57Bl/6 J background were maintained, unless otherwise stated, as separately bred colonies at the McGill University Health Center. Birc3 ${ }^{-/-38}$ and Ripk $3^{-/-73}$ have been previously described and Ripk $3^{-/-} /$Birc $^{-/-}$ were generated by crossbreeding. ${ }^{22}$ All animals used were 6-12-weekold male mice. All experiments were performed under guidelines of the animal ethics committee of McGill University (Canada).

Induction of tumorigenesis. Colitis-associated CRC was induced in mice by injecting intraperitoneally, on day 0, AOM (Sigma catalog (Cat) no. A2853, Oakville, Ontario, Canada) at a concentration of $10 \mathrm{mg} \mathrm{kg}^{-1}$. On day 7 , DSS $(55,000 \mathrm{kDa}$, MP Biomedical Cat no. 160110, Oakville, Ontario, Canada) was introduced in the drinking water at a concentration of $2 \%(w / v)$ for 1 week, and 2 weeks of regular drinking water followed. The DSS treatment was repeated for two additional cycles. Mice were killed on day 89; colons were removed from animals, flushed with cold PBS, and cut longitudinally. For the generation of bone marrow chimeras, WT and $B i r c 3^{-1-}$ mice were lethally irradiated (1,000 rads). The next day, mice were reconstituted with either WT or Birc3 ${ }^{-1-}$ bone marrow depleted of red blood cells. Chimerism was confirmed 6 weeks later, after which time point the AOM-DSS treatment was carried out. For the IL-18BP experiment, Birc3 ${ }^{-1-}$ mice were injected intraperitoneally with AOM $\left(10 \mathrm{mg} \mathrm{kg}^{-1}\right)$ on day 0 . On day 7 , mice were given $2 \%$ DSS in their drinking water for 5 days. During this time, IL-18BP was injected daily intraperitoneally at $20 \mu \mathrm{g} \mathrm{kg}^{-1}$.

Induction of colitis. Experimental colitis was induced by adding DSS (55 $000 \mathrm{kDa}$, MP Biomedical Cat no. 160110) to the drinking water at a concentration of $3 \%(\mathrm{w} / \mathrm{v})$. The animals were weighed daily and monitored for signs of distress as well as rectal bleeding. For acute induction of colitis, animals were treated for 5 days and then allowed to recover by drinking water for an additional 3 days. For chronic colitis, animals were treated for 5 days and then allowed to recover by drinking water for an additional 10 days. The cycle was repeated a second time and stopped after 3 days on regular water. For the SM experiments, LCL161 (Active Biomedical, Cat no. A-1147, Wanchai, Hong Kong) was orally gavaged beginning 6 days before DSS treatment and every 3 days until killing at a concentration of $50 \mathrm{mg} \mathrm{kg}^{-1}$ mouse bodyweight. DSS was added to the drinking water at a concentration of $3 \%(w / v)$ for 5 days followed by 3 days of regular drinking water. For the rescue experiments (IL-18, Nec-1, and zVAD-FMK), DSS was added to the drinking water at a concentration of $4 \%(\mathrm{w} / \mathrm{v})$ for 5 days followed by 3 days of regular water. For the IL-18 rescue experiments, murine recombinant IL-18 (R\&D, Cat no. B002-5, Minneapolis, MN) was injected intraperitoneally at a concentration of $0.05 \mu \mathrm{g}$ per mouse from days 0 to 4 after DSS. For the Nec-1 rescue experiment, Nec-1 (Sigma, Cat no. N9037) was injected intraperitoneally at a concentration of $5 \mathrm{mg} \mathrm{kg}^{-1}$ of bodyweight from days 0 to 4 after DSS. For the zVADFMK rescue experiment, zVAD-FMK (Bachem, Cat no. N1510, Budendorf, Switzerland) was injected intraperitoneally at a concentration of $3 \mathrm{mg} \mathrm{kg}^{-1}$ on days 0,2 , and 4 after DSS.

Hematoxylin and eosin staining and immunofluorescence. Colons were fixed in $10 \%$ buffered formalin overnight and paraffin embedded. Subsequently, $4 \mu \mathrm{m}$ thick sections were cut onto glass slides and processed for hematoxylin and eosin staining. Hematoxylin and eosin sections were scanned using ScanScope XT digital scanner (Leica, Concord, Ontario, Canada). ImageScope software (Leica) was used to quantify intestinal tissue damage and erosion by measuring the length of healthy, damaged, or fully eroded sections and each category expressed as a percentage of the full colon length. Healthy crypts were defined as organized crypts being unaffected from lumen to lamina propria, damaged crypts were defined as disorganized or incomplete crypts, and fully eroded sections were defined by the absence of crypts.

For the immunofluorescence, the slides were dewaxed with xylene twice for $5 \mathrm{~min}$. The sections were rehydrated in ethanol starting at $100 \%$, followed by 90,70 , and $50 \%$ ethanol for 5 min each. The slides were then incubated in water for $5 \mathrm{~min}$ and heated at $95^{\circ} \mathrm{C}$ for $15 \mathrm{~min}$ in a $0.1 \mathrm{~m}$ citrate buffer $(\mathrm{pH} 6.0)$ for antigen retrieval. PBS $0.05 \%$ Tween-20 was used to wash the slides. Slides were permeabilized with $0.25 \%$ Triton $\mathrm{X}-100$ in PBS for $20 \mathrm{~min}$ at room temperature. If required, Click-iT TUNEL Alexa Fluor 647 (Life Technologies, Cat no. C10247, Solon, Ontario, Canada) staining was performed according to the manufacturer's instructions. Slides were then blocked (10\% fetal bovine serum and $3 \%$ bovine serum albumin) for $30 \mathrm{~min}$ at $37^{\circ} \mathrm{C}$ and tissues were incubated with primary antibodies in PBS containing 3\% bovine serum albumin overnight at room temperature. The following antibodies were used: PCNA (Abcam, Cat no. AB2426, Toronto, Ontario, Canada), active caspse-3 (R\&D, Cat no. AF835), E-cadherin (BD Bioscience, Cat no. 610182, Mississauga, Ontario, Canada), F4/80 (eBioscience, Cat no. 45-4801-82, Burlington, Ontario, Canada), Gr-1 (BD, Cat no. 557661), CD19 (eBioscience, Cat no. 12-0191-81), CD3 (eBioscience, Cat no. 45-0031-82), and phospho-p65 (Cell Signaling, Cat no. 3033, Withby, Ontario, Canada). This step was followed by a 1$\mathrm{h}$ incubation with conjugated secondary antibodies coupled with Alexa Fluor 488, 594, or 647 (Invitrogen). The slides were rinsed with PBS and stained with Hoechst 33342 (Invitrogen, Cat no. H3570). Tissues were mounted with coverslips and analyzed on a Zeiss Axioskop upright wide-field microscope $(20 \times 0.5$ or $40 \times / 0.75$ Plan-Neofluar 
objectives) equipped with a high-resolution monochromatic AxioCam HRm camera and driven by AxioVision version 4.9.1 (Carl Zeiss Microscopy, Toronto, Ontario, Canada). ImageJ 1.46 (National Institute of Health, Boston, MA) was used for processing of entire images before cropping to emphasize the main point of the image when appropriate; processing was limited to background subtraction, brightness/contrast adjustments, and pseudo colors addition to facilitate the visualization/interpretation of the results. Quantification of proliferation and cell death was performed with the ImageJ Software on epifluorescence images cleared of background using the "BG subtraction from ROI" plugin. For proliferation, the numbers of PCNA-positive cells per well-oriented crypt were manually scored by visual inspection of images on screen. To quantify cell death, we used Volocity 6.0 (Quorum Technologies, Guelph, Ontario, Canada) to perform object-based analysis. Briefly, the crypt area was selected from epifluorescence images of colon sections stained with cell death markers (apoptosis: Active Caspase-3 (Red), late apoptosis and nonapoptotic cell death: TUNEL (FarRed)). Fluorescent objects (representing dead cells) in both channels were found by applying a set threshold and objects were delineated using a segmentation procedure based on a watershed algorithm. The number of active caspase-3-only, TUNEL-only, or double-positive cells (also positive for IEC marker E-cadherin) was determined and expressed as the number of epithelial cell death per $\mathrm{mm}^{2}$ of crypt area. Similarly, to quantify the number of phospho-p65-positive cells, fluorescent objects in both channels (red: phospho-p65-positive cells; blue: Hoechst/nucleus) were found and the number of phospho-p65-positive cells (cytoplasmic or nuclear) was determined. The results were stratified in a graph as phospho-p65positive cells per $\mathrm{mm}^{2}$ of tumor area. Images were collected and image processing and analysis for this manuscript was performed in the McGill University Life Sciences Complex Advanced BioImaging Facility.

Western blot. Tissues were lysed in buffer B150 (20 mM Tris-HCl, pH 8.0, $150 \mathrm{~mm} \mathrm{KCl}, 10 \%$ glycerol, $5 \mathrm{mM} \mathrm{MgCl}_{2}$, and $0.1 \% \mathrm{NP} 40$ ) supplemented with Complete-mini protease inhibitors (Roche Applied Science, Cat no. 11836153001, Laval, Quebec, Canada) and phosphatase inhibitors (Sigma Cat no. S7920, 71768, and G6376). Protein lysates were separated on sodium dodecyl sulfate-polyacrylamide gel electrophoresis and transferred to nitrocellulose membrane. Blots were probed with antibodies against Caspase-1 p20 (Genentech, San Francisco, CA), Caspase-3 (Cell Signaling, Cat no. 9661), Caspase-8 (Cell Signaling, Cat no. 9429), Caspase-11 (Sigma, Cat no. C1354), cFLIP (Cell Signaling, Cat no. 3210), cIAP1 (Enzo Life Sciences, Cat no. ALX-803-335-C100, Farmingdale, NY), IкB $\alpha$ (Cell Signaling, Cat no. 4812), NF-кB2 (Cell Signaling, Cat no. 4882), Phospo-Erk (Cell Signaling, Cat no. 9106), Total Erk (Cell Signaling, Cat no. 9102), Phospo-p38 (Cell Signaling, Cat no. 9211), Total p38 (Cell Signaling, Cat no. 9212), Rip3 (ProSci Incorporated, Cat no. 2283, Poway, CA), XIAP (R\&D, Cat no. AF8221), and $\beta$-actin (Sigma, Cat no. A1978).

Quantitative real-time PCR. Next, $2 \mu \mathrm{g}$ of total RNA was reverse transcribed with M-MLV reverse transcriptase (Invitrogen, Cat no. 28025-013) and random hexamers in a volume of $20 \mu \mathrm{l}$ according to the manufacturer's protocol. The primers used for quantitative realtime PCR are available upon request. All reactions were normalized to the housekeeping gene L32 to quantify the relative gene expression and were then analyzed using the $2^{-\Delta \Delta \mathrm{CT}}$ method.

Enzyme-linked immunosorbent assay and organ culture. Following DSS treatment, colons were removed from animals, flushed with cold PBS with penicillin and streptomycin, and cut longitudinally. A small piece $(0.5 \mathrm{~cm})$ of the colon was excised and washed twice more in clean PBS with penicillin streptomycin. The colon was cultured overnight in RPMI supplemented with glutamine, penicillin, streptomycin, and gentamicin. The supernatant was collected on the following morning and spun down to remove cellular debris. The following enzyme-linked immunosorbent assays were performed on the supernatant: IL-1 $\beta$ (R\&D, Cat no. DY401), IL-6 (R\&D, Cat no. DY406), IL-17 (R\&D, Cat no. DY421), and IL-18 (MBL International, Cat no. 1625, Woburn, MA).

Caspase-11 genotyping. DNA from ear notch was PCR amplified with primers Casp11-F-5'-AAGCAAAGAACCAATCAACA- $3^{\prime}$ and Casp11-R - $5^{\prime}$-TCAAAGAGATGACAAGAGCAAG-3' and the PCR product was purified using a QIAquick PCR Purification Kit (Quiagen, Cat no. 28106, Toronto, Ontario, Canada). The product was then digested for $1 \mathrm{~h}$ at $37^{\circ} \mathrm{C}$ with DPNII (BioLabs, Cat no. R0543, Withby, Ontario, Canada). The digestion was run on an agarose gel.

Statistical analysis. Data are represented as average \pm s.e. Two-tailed Student's $t$-test and analysis of variance were used for evaluating statistical significance between groups. Kaplan-Meier was used for survival analysis. ${ }^{\star} P<0.05 ;{ }^{* *} P<0.01 ;{ }^{\star * *} P<0.001$; NS, not significant.

SUPPLEMENTARY MATERIAL is linked to the online version of the paper at http://www.nature.com/mi

\section{ACKNOWLEDGMENTS}

We thank V. Dixit and Genentech for providing Ripk3 ${ }^{-1-}$ mice; R. Korneluk for Birc3 ${ }^{-1}$ Casp $11^{-1-}$ mice and caspase-11 PCR genotyping protocol; P. Barker for Birc $3^{-/-}$Casp $11^{+/+}$mice; J. Rinz and G. Perrault for animal husbandry. This work was supported by grants from the Canadian Institutes for Health Research (CIHR-MOP 82801) and the Burroughs Wellcome Fund to M.S. who is a Fonds de Recherche en Santé du Québec (FRSQ) Senior Investigator and a McGill University William Dawson Scholar. M.D., J.D. and A.S. are supported by doctoral studentships from the CIHR. A.M. was supported by a CIHR/CAG/Abbott fellowship.

\section{DISCLOSURE}

The authors declared no conflict of interest.

c) 2016 Society for Mucosal Immunology

\section{REFERENCES}

1. Asquith, M. \& Powrie, F. An innately dangerous balancing act: intestinal homeostasis, inflammation, and colitis-associated cancer. J. Exp. Med. 207, 1573-1577 (2010).

2. Dupaul-Chicoine, J., Dagenais, M. \& Saleh, M. Crosstalk between the intestinal microbiota and the innate immune system in intestinal homeostasis and inflammatory bowel disease. Inflamm. Bowel Dis. 19, 2227-2237 (2013).

3. Jostins, L. et al. Host-microbe interactions have shaped the genetic architecture of inflammatory bowel disease. Nature 491, 119-124 (2012).

4. Saleh, M. \& Trinchieri, G. Innate immune mechanisms of colitis and colitisassociated colorectal cancer. Nat. Rev. Immunol. 11, 9-20 (2011).

5. Dagenais, M., Douglas, T. \& Saleh, M. Role of programmed necrosis and cell death in intestinal inflammation. Curr. Opin. Gastroenterol. 30, 566-575 (2014).

6. Neurath, M.F. Cytokines in inflammatory bowel disease. Nat. Rev. Immunol. 14, 329-342 (2014).

7. Darding, M. \& Meier, P. IAPs: guardians of RIPK1. Cell Death Differ. 19, 58-66 (2012).

8. Bertrand, M.J. et al. clAP1 and clAP2 facilitate cancer cell survival by functioning as E3 ligases that promote RIP1 ubiquitination. Mol. Cell 30, 689-700 (2008).

9. Dannappel, M. et al. RIPK1 maintains epithelial homeostasis by inhibiting apoptosis and necroptosis. Nature 513, 90-94 (2014).

10. Takahashi, N. et al. RIPK1 ensures intestinal homeostasis by protecting the epithelium against apoptosis. Nature 513, 95-99 (2014).

11. Vandenabeele, P., Galluzzi, L., Vanden Berghe, T. \& Kroemer, G. Molecular mechanisms of necroptosis: an ordered cellular explosion. Nat. Rev. Mol. Cell Biol. 11, 700-714 (2010).

12. Kaiser, W.J. et al. RIP3 mediates the embryonic lethality of caspase8-deficient mice. Nature 471, 368-372 (2011). 
13. Oberst, A. et al. Catalytic activity of the caspase-8-FLIP(L) complex inhibits RIPK3-dependent necrosis. Nature 471, 363-367 (2011).

14. Varfolomeev, E.E. et al. Targeted disruption of the mouse caspase 8 gene ablates cell death induction by the TNF receptors, Fas/Apo1, and DR3 and is lethal prenatally. Immunity 9, 267-276 (1998).

15. Yeh, W.C. et al. FADD: essential for embryo development and signaling from some, but not all, inducers of apoptosis. Science 279, 1954-1958 (1998).

16. Yeh, W.C. et al. Requirement for Casper (c-FLIP) in regulation of death receptor-induced apoptosis and embryonic development. Immunity $\mathbf{1 2}$, 633-642 (2000).

17. Zhang, H., Zhou, X., McQuade, T., Li, J., Chan, F.K. \& Zhang, J. Functional complementation between FADD and RIP1 in embryos and lymphocytes. Nature 471, 373-376 (2011).

18. Gunther, C. et al. Caspase-8 regulates TNF-alpha-induced epithelial necroptosis and terminal ileitis. Nature 477, 335-339 (2011).

19. Weinlich, R. et al. Protective roles for caspase-8 and cFLIP in adult homeostasis. Cell Rep. 5, 340-348 (2013).

20. Welz, P.S. et al. FADD prevents RIP3-mediated epithelial cell necrosis and chronic intestinal inflammation. Nature 477, 330-334 (2011).

21. Linkermann, A. \& Green, D.R. Necroptosis. N. Engl. J. Med. 370, 455-465 (2014).

22. Rodrigue-Gervais, I.G. et al. Cellular inhibitor of apoptosis protein CIAP2 protects against pulmonary tissue necrosis during influenza virus infection to promote host survival. Cell Host Microbe 15, 23-35 (2014).

23. Wallach, D., Kovalenko, A. \& Kang, T.B. 'Necrosome'-induced inflammation: must cells die for it? Trends Immunol. 32, 505-509 (2011).

24. Kang, T.B., Yang, S.H., Toth, B., Kovalenko, A. \& Wallach, D. Activation of the NLRP3 Inflammasome by Proteins That Signal for Necroptosis. Methods Enzymol. 545, 67-81 (2014).

25. Gurung, P. etal. FADD and caspase-8 mediate priming and activation of the canonical and noncanonical Nirp3 inflammasomes. J. Immunol. 192, 1835-1846 (2014).

26. Moulin, M. et al. IAPs limit activation of RIP kinases by TNF receptor 1 during development. EMBO J. 31, 1679-1691 (2012).

27. Bertrand, M.J., Doiron, K., Labbe, K., Korneluk, R.G., Barker, P.A. \& Saleh, M. Cellular inhibitors of apoptosis cIAP1 and CIAP2 are required for innate immunity signaling by the pattern recognition receptors NOD1 and NOD2. Immunity 30, 789-801 (2009).

28. Tseng, P.H., Matsuzawa, A., Zhang, W., Mino, T., Vignali, D.A. \& Karin, M. Different modes of ubiquitination of the adaptor TRAF3 selectively activate the expression of type I interferons and proinflammatory cytokines. Nat. Immunol. 11, 70-75 (2010).

29. Labbe, K., Mclntire, C.R., Doiron, K., Leblanc, P.M. \& Saleh, M. Cellular inhibitors of apoptosis proteins CIAP1 and CIAP2 are required for efficient caspase-1 activation by the inflammasome. Immunity 35 , 897-907 (2011).

30. Petersen, S.L. et al. Autocrine TNFalpha signaling renders human cancer cells susceptible to Smac-mimetic-induced apoptosis. Cancer Cell 12, 445-456 (2007).

31. Varfolomeev, E. et al. IAP antagonists induce autoubiquitination of C-IAPs, NF-kappaB activation, and TNFalpha-dependent apoptosis. Cell 131, 669-681 (2007).

32. Vince, J.E. et al. IAP antagonists target CIAP1 to induce TNFalphadependent apoptosis. Cell 131, 682-693 (2007).

33. Beug, S.T. et al. Smac mimetics and innate immune stimuli synergize to promote tumor death. Nat. Biotechnol. 32, 182-190 (2014).

34. Seidelin, J.B., Vainer, B., Andresen, L. \& Nielsen, O.H. Upregulation of CIAP2 in regenerating colonocytes in ulcerative colitis. Virchows Arch. 451, 1031-1038 (2007)

35. Karasawa, H. et al. Down-regulation of clAP2 enhances 5-FU sensitivity through the apoptotic pathway in human colon cancer cells. Cancer Sci. 100, 903-913 (2009).

36. Krajewska, M. et al. Analysis of apoptosis protein expression in early-stage colorectal cancer suggests opportunities for new prognostic biomarkers. Clin. Cancer Res. 11, 5451-5461 (2005).

37. Miura, K. et al. Inhibitor of apoptosis protein family as diagnostic markers and therapeutic targets of colorectal cancer. Surg. Today 41, 175-182 (2011).
38. Conte, D. et al. Inhibitor of apoptosis protein clAP2 is essential for lipopolysaccharide-induced macrophage survival. Mol. Cell. Biol. 26, 699-708 (2006).

39. Kayagaki, N. et al. Non-canonical inflammasome activation targets caspase-11. Nature 479, 117-121 (2011).

40. Kenneth, N.S. et al. An inactivating caspase 11 passenger mutation originating from the 129 murine strain in mice targeted for C-IAP1. Biochem. J. 443, 355-359 (2012).

41. Gyrd-Hansen, M. \& Meier, P. IAPs: from caspase inhibitors to modulators of NF-kappaB, inflammation and cancer. Nat. Rev. Cancer 10, 561-574 (2010).

42. Gonzalez-Lopez, M. et al. Design, synthesis and evaluation of monovalent Smac mimetics that bind to the BIR2 domain of the anti-apoptotic protein XIAP. Bioorg. Med. Chem. Lett. 21, 4332-4336 (2011).

43. Huber, S. et al. IL-22BP is regulated by the inflammasome and modulates tumorigenesis in the intestine. Nature 491, 259-263 (2012).

44. Allen, I.C. et al. The NLRP3 inflammasome functions as a negative regulator of tumorigenesis during colitis-associated cancer. J. Exp. Med. 207, 1045-1056 (2010).

45. Dupaul-Chicoine, J. et al. Control of intestinal homeostasis, colitis, and colitis-associated colorectal cancer by the inflammatory caspases. Immunity 32, 367-378 (2010).

46. Elinav, E. et al. NLRP6 inflammasome regulates colonic microbial ecology and risk for colitis. Cell 145, 745-757 (2011).

47. Salcedo, R. et al. MyD88-mediated signaling prevents development of adenocarcinomas of the colon: role of interleukin 18. J. Exp. Med. 207, 1625-1636 (2010).

48. Wlodarska, M. et al. NLRP6 inflammasome orchestrates the colonic hostmicrobial interface by regulating goblet cell mucus secretion. Cell 156, 1045-1059 (2014).

49. Zaki, M.H., Boyd, K.L., Vogel, P., Kastan, M.B., Lamkanfi, M. \& Kanneganti, T.D. The NLRP3 inflammasome protects against loss of epithelial integrity and mortality during experimental colitis. Immunity 32, 379-391 (2010a).

50. Demon, D., Kuchmiy, A., Fossoul, A., Zhu, Q., Kanneganti, T.D. \& Lamkanfi, M. Caspase-11 is expressed in the colonic mucosa and protects against dextran sodium sulfate-induced colitis. Mucosal Immunol. 7 , 1480-1491 (2014).

51. Oficjalska, K. et al. Protective role for caspase-11 during acute experimental murine colitis. J. Immunol. 194, 1252-1260 (2015).

52. Grivennikov, S. et al. IL-6 and Stat3 are required for survival of intestinal epithelial cells and development of colitis-associated cancer. Cancer Cell 15, 103-113 (2009).

53. Putoczki, T.L. et al. Interleukin-11 is the dominant IL-6 family cytokine during gastrointestinal tumorigenesis and can be targeted therapeutically. Cancer Cell 24, 257-271 (2013).

54. Feoktistova, M. et al. clAPs block Ripoptosome formation, a RIP1/caspase-8 containing intracellular cell death complex differentially regulated by cFLIP isoforms. Mol. Cell 43, 449-463 (2011).

55. Tenev, T. et al. The Ripoptosome, a signaling platform that assembles in response to genotoxic stress and loss of IAPs. Mol. Cell 43, 432-448 (2011).

56. Vanden Berghe, T., Linkermann, A., Jouan-Lanhouet, S., Walczak, H. \& Vandenabeele, P. Regulated necrosis: the expanding network of nonapoptotic cell death pathways. Nat. Rev. Mol. Cell Biol. 15, 135-147 (2014).

57. Duprez, L., Bertrand, M.J., Vanden Berghe, T., Dondelinger, Y., Festjens, N. \& Vandenabeele, P. Intermediate domain of receptor-interacting protein kinase 1 (RIPK1) determines switch between necroptosis and RIPK1 kinase-dependent apoptosis. J. Biol. Chem. 287, 14863-14872 (2012).

58. Basu, R. et al. IL-1 signaling modulates activation of STAT transcription factors to antagonize retinoic acid signaling and control the $\mathrm{TH} 17$ cell-iTreg cell balance. Nat. Immunol. 16, 286-295 (2015).

59. Zaki, M.H., Vogel, P., Body-Malapel, M., Lamkanfi, M. \& Kanneganti, T.D. IL-18 production downstream of the Nlrp3 inflammasome confers protection against colorectal tumor formation. J. Immunol. 185, 4912-4920 (2010b).

60. Greten, F.R. et al. IKKbeta links inflammation and tumorigenesis in a mouse model of colitis-associated cancer. Cell 118, 285-296 (2004).

61. Qiu, W. et al. An apoptosis-independent role of SMAC in tumor suppression. Oncogene 32, 2380-2389 (2013). 
62. Damgaard, R.B. et al. Disease-causing mutations in the XIAP BIR2 domain impair NOD2-dependent immune signalling. EMBO Mol. Med. 5, 1278-1295 (2013).

63. Filipovich, A.H., Zhang, K., Snow, A.L. \& Marsh, R.A. X-linked lymphoproliferative syndromes: brothers or distant cousins? Blood 116, 3398-3408 (2010).

64. Marsh, R.A. et al. Allogeneic hematopoietic cell transplantation for XIAP deficiency: an international survey reveals poor outcomes. Blood 121, 877-883 (2013).

65. Pachlopnik Schmid, J. et al. Clinical similarities and differences of patients with X-linked lymphoproliferative syndrome type 1 (XLP-1/SAP deficiency) versus type 2 (XLP-2/XIAP deficiency). Blood 117, 1522-1529 (2011).

66. Rigaud, S. et al. XIAP deficiency in humans causes an X-linked lymphoproliferative syndrome. Nature 444, 110-114 (2006).

67. Speckmann, C. et al. X-linked inhibitor of apoptosis (XIAP) deficiency: the spectrum of presenting manifestations beyond hemophagocytic lymphohistiocytosis. Clin. Immunol. 149, 133-141 (2013).
68. Yang, X. et al. Clinical and genetic characteristics of XIAP deficiency in Japan. J. Clin. Immunol. 32, 411-420 (2012).

69. Zeissig, Y. et al. XIAP variants in male Crohn's disease. Gut 64, 66-76 (2014).

70. Nenci, A. et al. Epithelial NEMO links innate immunity to chronic intestinal inflammation. Nature 446, 557-561 (2007).

71. Qiu, W. et al. PUMA-mediated intestinal epithelial apoptosis contributes to ulcerative colitis in humans and mice. J. Clin. Invest. 121, 1722-1732 (2011).

72. Qiu, W., Carson-Walter, E.B., Kuan, S.F., Zhang, L. \& Yu, J. PUMA suppresses intestinal tumorigenesis in mice. Cancer Res. 69, 4999-5006 (2009).

73. Newton, K., Sun, X. \& Dixit, V.M. Kinase RIP3 is dispensable for normal NF-kappa Bs, signaling by the B-cell and T-cell receptors, tumor necrosis factor receptor 1, and Toll-like receptors 2 and 4. Mol. Cell. Biol. 24, 1464-1469 (2004). 